\title{
OPTIMISATION OF MAGNETS POSITIONS ON THE KNEE JOINT USING COMSOL MULTIPHYSICS
}

\author{
LAPTOIU, D[an] C[onstantin]; ANTONIAC, I[ulian] \& COTRUT, C[osmin]
}

\begin{abstract}
The purpose of the project presented in this paper consists in the development of a multifunctional knee device to be used in the early stages of treatment of osteoarthritis. Magnetically controlled vascosupplimentation therapy is a new concept of bioactive treatment, which combines the advantages of intra-articular therapy assisted by a more complex system of fixing and controlling joint mobility. Several positions and dimensions of magnets on two different knee positions (flexion/extension) were analysed and optimised using finite elements analysis software, in order to create a magnetic field able to localize and concentrate the nanoparticles for drugs delivery.
\end{abstract}

Key words: finite elements analysis, magnetic controlled therapy, nanoparticles, magnetic field optimisation

\section{INTRODUCTION}

The main goals in treating degenerative knee joint disease (such as arthritis) are minimizing the pain, maintaining or improving joint mobility and minimizing functional deterioration. Infiltration of the knee with steroids is in current medical practice for over 50 years and vascosupplimentation for over 10 years. A newer approach is magnetically controlled vascosupplimentation, which aims to restore the viscoelastic qualities of the joint fluid, with concomitant improvement of rheology as a result of endogenous synthesis and normalization of accelerated degradation of hyaluronate into the joint. This therapy requires the use of an orthosis with a specialized anatomic design, modified to allow insertion of groups of permanent magnets for combined use with application of infiltration of a composite viscoelastic biopolymer - magnetic nanoparticles.

The research was focused on two main objectives: 1. Development of magnetic nanoparticles for correcting osteoarthritic knees. Magnetite nanoparticles with $20-30 \mathrm{~mm}$ dimensions, coated with amine for controlling the aggregation during synthesis were obtained, and tests with different concentration were performed.

2. Design and manufacturing a knee orthosis with magnets for enhancing drugs action time in the interest area. Several cases with different magnets dimensions and placements were analysed using Comsol Multiphysics simulation software for optimizing their position by taking into account the influence on the magnetic field. The approach presented in the current paper considers first the use of one magnet with dimension of $10 \times 2 \mathrm{~mm}$, and then arrangements of 2, 3 and 4 magnets spread on a surface of $10 \mathrm{~mm}^{2}$ - all magnets were neodymium iron boron, $1220-1250 \mathrm{mT}$ and $287 \mathrm{~kJ} / \mathrm{m}^{3}$ maximal energy.

Analysis of the literature showed that magnetic orthoses currently used are based on the effect of the magnetic field properties for pain relief (Chen, 2008), (Mendel et al., 2010) and do not consider the combined action of magnetic nanoparticles infiltrated in the interest zone and the magnetic field created by magnets.
Moreover, the field of orthosis design is focused more on developing new technical solutions (Chen \& Liao, 2009) and (Nikitczuk et al., 2004) for improving existent designs.

\section{MAGNETIC NANOPARTICLES FOR IMPROVING DRUGS DELIVERY}

The magnetic assisted intra-articular therapy (hyaluronate covered magnetic nanoparticles with viscoelastic properties) is focused on the articular defect; the effect must be maintained for at least three weeks. (Mitsuo et al., 2001) presented a similar solution based therapeutics and cultures of chondrocytes atelocolagen. Nanoparticles used in attempts to correct this deficiency, will be loaded into a proposed concentration between 10 and $200 \mathrm{mg} / \mathrm{ml}$ to increase the biomechanical properties. Also the concentration expressed in $\mathrm{mg}$ per $\mathrm{mg}$ hyaluronic acid product will be different, ranging from 8 to 15 between different preparations and the amount is about $5 \mathrm{ml}$.

Since the particles are scattered in the synovial liquid, the recognition and elimination by phagocytes can take a long time. Unlike inorganic magnetic particles, biodegradable polymers may release the active absorbed ingredient in a controlled way by determining their rate of degradation (Cinteza et al., 2006), (Gijs, 2010).

The use of magnetic core particles and biodegradable polymer shell can take advantage of the properties of the two components. Thus, the magnetic core particles can transport you directly to the target and keep it there by applying an external magnetic field. Detailed studies (Morega, 2006), (Lee\&Shin, 2007), (Butoescu, 2010) have established that magnetic carriers must have the following properties: a near field magnetic gradients and flow of the body's physiological system, the ability to carry a larger amount. The active ingredient, not excessively load the body with magnetic material, speed controllable release the active compound in the target area, properties that provide maximum surface biocompatibility, biodegradability and allowing easy elimination of minimizing toxicity in the body.

\section{MAGNETIC FIELD OPTIMISATION FOR A KNEE ORTHOSIS}

COMSOL Multiphysics software was used for assessing the magnetic field magnitude for nine cases obtained by varying the ratio between magnet length and distance between magnets (d1/d2) and positions matrices, as it can be seen in figures 2-3.

Figure 1 presents several results of the experiments for the case in which the ratio $\mathrm{d} 1 / \mathrm{d} 2<1$ (for 1 magnet, $2 \times 2$ magnets, $3 \times 3$ magnets, $4 \times 4$ magnets), along an illustration of the directions in which the ferrofluid placed in the intra-articular space will move when subjected to the magnetic field action.

Similar analyses were performed for all cases; results comparison allowed determining the best position of magnets on the analysed patello-femural knee orthosis. 


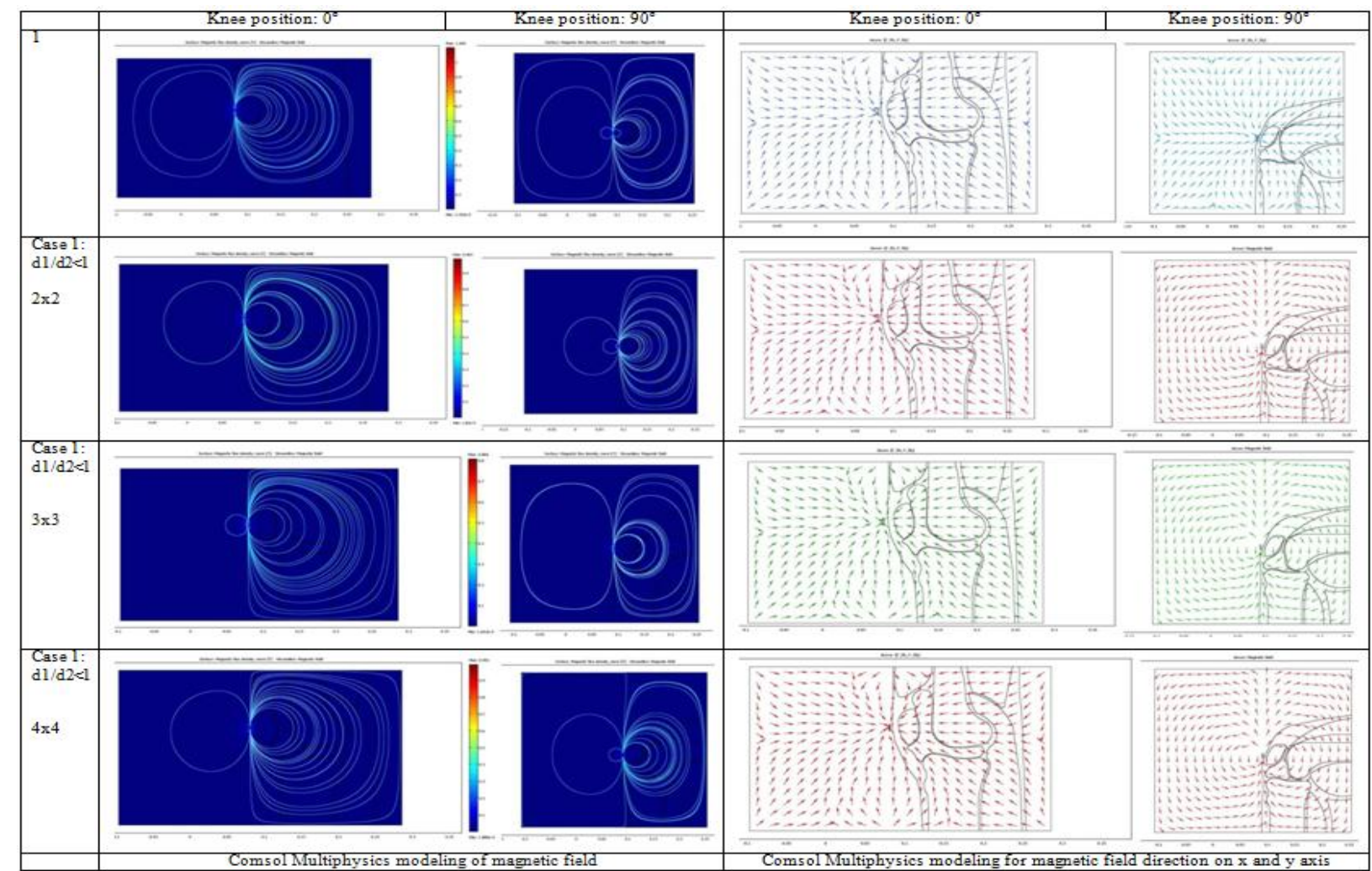

Fig.1. Comsol Multiphysics analysis of knee orthosis magnets position influence on the magnetic field

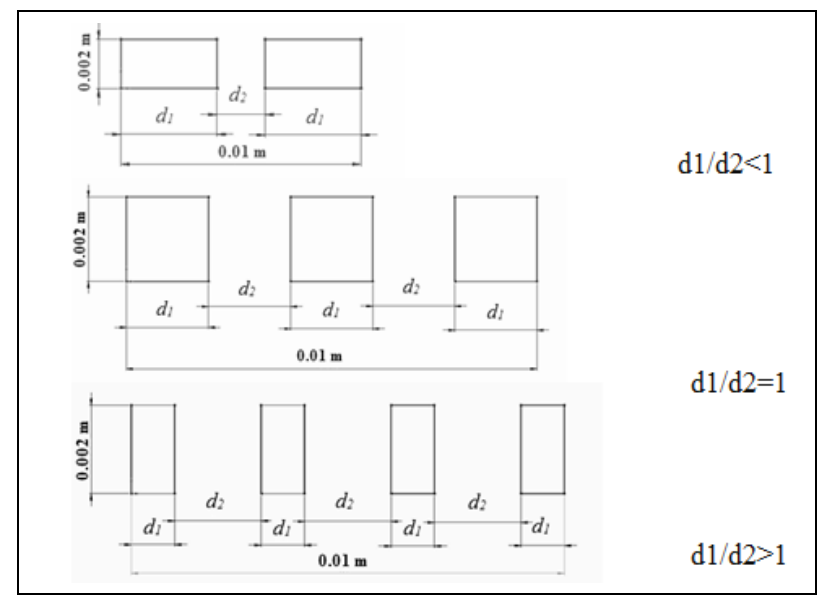

Fig.2. Magnets matrices for 2D modelling

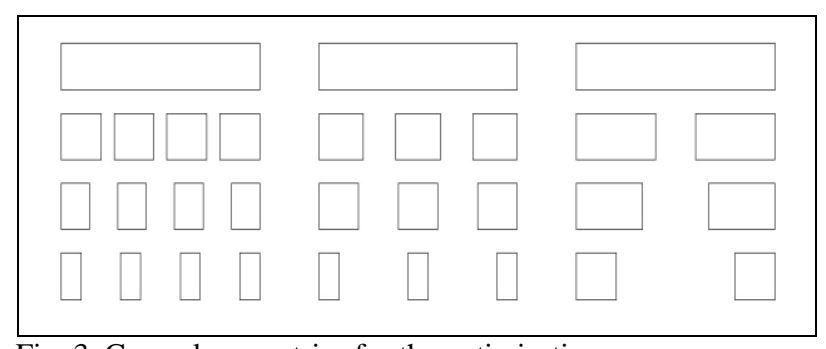

Fig. 3. Comsol geometries for the optimisation process

\section{CONCLUSIONS}

The main conclusion of the analysis performed was that in order to obtain the best results in drugs delivery in the patellofemural targeted area, an arrangement of $4 \times 4$ magnets on a square pattern close to the rotula at distances which satisfy condition $\mathrm{d} 1 / \mathrm{d} 2<1$ for ensuring a larger concentration of the magnetic nanoparticles.

These results are confirmed by the experiments performed in the project and which will be presented in further articles.

\section{ACKNOWLEDGEMENTS}

This work was supported by CNCSIS, PNCDI2, Project 42132, ARTROMAG - Medical device for joint articular disease therapy using nanomaterials and magnetic field effects.

\section{REFERENCES}

Chen, C.I. et al., (2008), Effect of Magnetic Knee Wrap on Quadriceps Strength in Patients With Symptomatic Knee Osteoarthritis, Archives of Physical Medicine and Rehabilitation, Volume 89, Issue 12, pp. 2258 - 2264

Mendel, et al., (2010), Magnetic isolation of particles suspended in synovial fluid for diagnostics of natural joint chondropathies, Acta Biomaterialia, 6, pp.4430-4438

Chen, J., Liao, W.H., (2009), Design, testing and control of a magnetorheological actuator for assistive knee braces, Smart Mater. Struct., vol.19, no.3, pp.029-035

Butoescu, N., et al. (2009). Dexamethasone-containing biodegradable superparamagnetic microparticles for intraarticular administration: physicochemical and magnetic properties, in vitro and in vivo drug release. Eur J Pharm Biopharm, 72(3), pp.529-38.

Mitsuo, O. et al. (2001). Current Concepts in Tissue Engineering Technique for Repair of Cartilage Defect, Arificial Organs, Volume 25, Issue 3, pp.172-179

Cintez, L.O., et al., (2006). Diacyllipid micelle-based nanocarrier for magnetically guided delivery of drugs in photodynamic therapy. Mol. Pharm., 3, pp. 415-423

Gijs, M.A.M., (2010). Microfluidic applications of magnetic particles for biological analysis and catalysis, Chem. Rev. 2010, 110, 1518-1563

Morega, A.M. et al. (2005). A FEM analysis of magnetically induced biomagnetic fluid mixing, Rev. Roum. Scie. Tech. Electrotechnique et Energetique, 50, 2, pp.239-248

Lee, S.H., Shin, H. (2007). Matrices and scaffolds for delivery of bioactive molecules in bone and cartilage tissue engineering. Advanced Drug Delivery Rev., 59 pp.339-35 